Revista de Comunicación y Salud, 2017, Vol. 7, pp. 103-117

Editado por Cátedra de Comunicación y Salud

ISSN: 2174-5323 (impreso) 2173-1675 (electrónico)

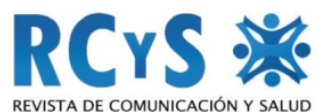

Enviado 22 de septiembre de 2017 Aprobado 15 de noviembre de 2017

\title{
AMBIENTE LIMPIO, COMUNIDAD FELIZ: ANÁLISIS DE RESULTADOS DE UNA CAMPAÑA DE COMUNICACIÓN PARA LA PREVENCIÓN DE LA FIEBRE DEL DENGUE EN LAS COMUNIDADES LUIS XV, EN GUÁPILES, COSTA RICA ${ }^{1}$
}

\author{
Clean Environment, Happy Community. Analysis of a communication \\ campaign for Dengue prevention in Luis XV, in Guaplites, Costa Rica. \\ Yanet Martínez Toledo ${ }^{2}$, Lissette Marroquin \\ Centro de Investigación en Comunicación. Universidad de Costa Rica
}

\section{Resumen}

Este artículo presenta los resultados de la evaluación de la campaña de comunicación para la prevención del dengue "Ambiente Limpio, Comunidad Feliz" que se implementó del 27 de septiembre al 6 de diciembre de 2014 en la comunidad de Luis XV, en la Roxana de Guápiles. La campaña es el resultado de un proceso de investigación ${ }^{3}$ que inicia en marzo de 2012 y que se caracterizó por la participación activa de ciertos grupos de vecinos y vecinas de las comunidades no sólo en la investigación sino también en el diseño e implementación de la campaña. El propósito del artículo es identificar los beneficios y las limitaciones de este tipo de iniciativas para el desarrollo e implementación de campañas de comunicación para la prevención del dengue. Para ello se presentan los antecedentes de la campaña, una descripción de la misma y la posterior evaluación de resultados.

Palabras clave: campaña de comunicación, evaluación del impacto de campañas de prevención, investigación acción participativa (IAP), investigación participativa basada en la comunidad (IPbC).

\footnotetext{
Abstract

In this paper the authors present results evaluation of a Communication Campaign "Clean Environment, Happy Community" to prevent Dengue Disease in Luis XV, located in Guápiles, Costa Rica, in 2014. This campaign is the result of a participatory research process ${ }^{4}$, started in March 2012. As researchers, we want to highlight the active participation of neighbors, as one of the main results of the process. The main goal of this paper is to identify the benefits and limitation of

${ }^{1}$ Los datos que se presentan en este artículo son producto del trabajo del equipo de investigación de la Universidad de Costa Rica compuesto por las siguientes personas: Adrián Avendaño, Lissette Marroquín, Yanet Martínez, Carlos Murcia y Elsy Vargas.

${ }^{2}$ Autor para contacto: yanet.martinez_t@ucr.ac.cr

${ }^{3} 4$ En el marco del proyecto 835-B2510 "Fomento a la capacidad para investigaciones basadas en la participación de la comunidad dirigidas hacia campañas de salud pública para la prevención y control de la epidemia de dengue en la provincia de Limón, Costa Rica" inscrito en el Centro de Investigaciones en Comunicación (CICOM) de la Universidad de Costa Rica.
} 
Ambiente limpio, comunidad feliz: análisis de resultados de una campaña de comunicación para la prevención de la fiebre del dengue en las comunidades Luis XV, en Guápiles, Costa Rica

Participatory Research and the development of a Campaign of Dengue prevention based on this process. That's why, we describe and evaluate the Campaign.

Key words: Communication campaign, campaign's impact, Participatory Research, Participatory Research based in Community.

Para citar el artículo

Martínez Toledo, Y., Marroquin, L. (2017). Ambiente limpio, comunidad feliz: análisis de resultados de una campaña de comunicación para la prevención de la fiebre del dengue en las comunidades Luis XV, en Guápiles, Costa Rica. Revista de Comunicación y Salud, vol. 7, 103-117. Recuperado de http://revistadecomunicacionysalud.org/index.php/rcys/article/view/127

\section{INTRODUCCIÓN}

En este artículo presentamoss un análisis de los resultados de la evaluación de la campaña "Ambiente Limpio, Comunidad Feliz" que se implementa en Luis XV, en Guápiles entre el 27 de setiembre y el 6 de diciembre del 2014. En este texto, por cuestiones de espacio, solo se analizan los resultados obtenidos en la comunidad de Luis XV. El texto está organizado de la siguiente manera. Primeramente, ofrecemos una serie de datos que permiten contextualizar la campaña. Luego describimos el proceso de diseño e implementación de la campaña para finalmente realizar una evaluación de la misma.

\section{Antecedentes de la campaña}

\subsection{Un proyecto de investigación interdisciplinario e interuniversitario}

La campaña "Ambiente Limpio, Comunidad Feliz" se inserta dentro de un proceso de investigación más amplio que inicia en marzo 2012 con la inscripción del proyecto "Fomento a la capacidad para investigaciones basadas en la participación de la comunidad dirigidas hacia campañas de salud pública para la prevención y control de la epidemia de dengue en la provincia de Limón, Costa Rica". Se trata de un proyecto de investigación interdisciplinario que involucra a las escuelas de Comunicación y Salud Pública de la Universidad de Costa Rica (UCR) y sus homólogas en la Universidad de Kansas (KU). El proyecto se plantea como objetivo el desarrollo de una campaña piloto para la prevención del dengue a partir de la metodología de Investigación participativa basada en la comunidad (IPbC).

La temática del dengue despierta interés en las cuatro unidades académicas por tratarse de una enfermedad de gran incidencia a nivel global. Según Shepard, Coudeville, Halasa, Zambrano, y Dayan (2011), a la fecha, el dengue es una de las epidemias más retadoras de América, incurriendo en serias cargas humanas y económicas. Año tras año las variantes de la epidemia son significativas, se estima que el impacto económico de esta enfermedad podría llegar a los 2.1 millones de dólares en promedio al año (Shepard et al., 2011).

Si bien el dengue puede ser mortal, se trata de una enfermedad prevenible cuya propagación está íntimamente relacionada con las prácticas cotidianas de las personas en comunidad. "Los criaderos del Aedes aegypti están muy relacionados 
con factores ecológicos macro y micro que son determinados por los comportamientos humanos -individuales, colectivos e institucionales- y sus contextos social, económico y político" (Quintero et al., 2014, p. 2). En otras palabras, la dimensión cultural y social inciden directamente en la dinámica de transmisión de la enfermedad en el espacio comunitario. Por ende el desarrollo de acciones para la prevención del dengue deben articular conocimientos no sólo de salud pública y de promoción de la salud sino también de ciencias sociales y particularmente a la comunicación que permite explorar la construcción de sentido (dimensión cultural y social) de individuos y comunidades.

El primer paso en el proceso fue la selección de las comunidades donde se realizaría el proyecto piloto. Para la selección se establecen varios criterios, entre los cuales destaca la incidencia de casos. En el momento en que inicia el proyecto, la región Huetar Atlántica presenta la mayor incidencia de casos de dengue. El cantón de Pococí, el más poblado con 125,962 habitantes (INEC, 2011), es el segundo cantón más afectado por la epidemia del dengue con 164 casos reportados en el 2011 (División de Vigilancia de la Salud, Pococí, 2011), 683 en el 2013 y 228 a la semana 24 del 2014, (Memoria Institucional 2010-2014, p. 70). Se selecciona el distrito de La Roxana para el proyecto por la alta incidencia de casos de dengue, por sus características socio demográficas y por qué cuenta con algunos grupos comunitarios organizados.

A continuación, se resumen las principales fases del proyecto de investigación donde se inserta la campaña.

1. Mapeo de actores comunitarios: a partir de entrevistas con funcionarios de instituciones públicas y otras organizaciones con presencia en la localidad, se realiza un mapeo de actores para identificar líderes comunales.

2. Primer contacto: se realizan entrevistas informales con las y los líderes de ambas comunidades que se identificaron en el mapeo de actores. Se valora el interés que tienen de participar en el proyecto y de convocar a otras personas de la comunidad.

3. Diagnóstico participativo: se convoca a un taller para identificar cuáles son los problemas más importantes que afectan a cada comunidad. En el diagnóstico se destaca: el mal manejo de residuos sólidos, la escasez de oportunidades laborales, la delincuencia y la drogadicción.

4. Establecimiento de una agenda de trabajo: se trabaja con las y los participantes para priorizar problemas y para determinar cuáles de estos podrían de ser atendidos a través de un plan de comunicación. Se acuerda trabajar en el desarrollo de conocimientos para mejorar las prácticas de manejo de residuos sólidos. Este es un tema que se articula con la prevención del dengue.

5. Encuesta para la línea base: se elabora un cuestionario de Conocimientos, Actitudes y Percepciones (CAP) acerca del dengue.

6. Fomento a la capacidad de organización comunitaria: se inicia una serie de talleres que tienen por objetivo el fortalecer las capacidades de las y los participantes para que puedan asumir un papel más fuerte en la elaboración del plan de comunicación para la prevención del dengue y la implementación de la 
Ambiente limpio, comunidad feliz: análisis de resultados de una campaña de comunicación para la prevención de la fiebre del dengue en las comunidades Luis XV, en Guápiles, Costa Rica

campaña. Se desarrolla el tema del reciclaje con énfasis en transformación de residuos sólidos en objetos de uso cotidiano. También se trabaja el tema de la comunicación asertiva y el liderazgo.

7. Diseño de la campaña: en este proceso participan las agentes comunitarias y representantes de las instituciones públicas relacionadas con los temas de dengue y el manejo de residuos sólidos. Se realizan cuatro sesiones donde representantes de las instituciones y miembros de la comunidad convergieron para el establecimiento de acciones conjuntas en la campaña "Ambiente Limpio, Comunidad Feliz".

8. Implementación de la campaña: La campaña se concentra en la eliminación de posibles criaderos del mosquito del dengue a partir de un manejo adecuado de residuos sólidos en los hogares. Para ello, se coordina un sistema gratuito de recolección de residuos reciclables. El servicio se complementa con los Talleres de "Mi casa verde" espacios de co-aprendizaje sobre reciclaje, eliminación o transformación de criaderos y recuperación de residuos por medio de manualidades.

9. Evaluación: El proceso de investigación y la campaña de comunicación se evalúa en términos de su alcance e impacto. Para ello, se administra una encuesta de Conocimientos, Actitudes y prácticas en torno a la prevención del dengue antes y después de la implementación de la campaña.

\subsection{Elecciones metodológicas y principios de trabajo}

La propuesta metodológica tuvo como antecedentes las experiencias de Investigación Acción Participativa (IAP) realizadas tanto en la Escuela de Salud Pública como la Escuela de Comunicación de la UCR y las experiencias en Investigación participativa basada en la comunidad (IPbC) del Departamento de Medicina Familiar de Kansas University. Se trata de dos acercamientos metodológicos muy similares que como lo manifiestan los autores Lazarus, Duran, Caldwell y Bulbulia (2012) más que instrumentación para investigación constituyen formas de "ver y entender el mundo (worldviews)". Ambas metodologías comparten a) una visión de la comunidad como unidad de identidad, de soluciones y de práctica; b) el involucramiento de la comunidad en todas las fases del proceso de investigación; c) el establecimiento claro de los beneficios; y d) el co-aprendizaje entre los participantes (p. 311).

En la década de los setenta, e inspirados en la primera versión de investigación acción (Lewin, 1946), desde América Latina surge, con el trabajo de investigación desarrollado por Fals Borda, una corriente de investigación acción participativa (Fals Borda, Bonilla y Castillo, 1972). El método propuesto se basaba en la inserción del equipo de investigación en la comunidad, el análisis de las condiciones históricas y estructura social de la comunidad, el desarrollo de un nivel de conciencia sociopolítica de las personas de la comunidad, el desarrollo de grupos de acción.

Si bien la mayoría de estos principios son también la base para la metodología $\mathrm{IPbC}$, la dimensión política de la investigación y su papel en el cambio social son los principales aportes de la IAP planteada por Fals Borda. Con sus raíces puestas en la propuesta teórico-metodológica de Educación Popular planteada por Paulo Freire 
Ambiente limpio, comunidad feliz: análisis de resultados de una campaña de comunicación para la prevención de la fiebre del dengue en las comunidades Luis XV, en Guápiles, Costa Rica

(1970), la IAP coloca el proceso de investigación en un contexto comunitario donde el "desarrollo de conciencia crítica entre los participantes, se convierte en un proceso liberador" (Balcazar, 2003, p. 62). Al participar activamente en los procesos de investigación-acción (diagnóstico de problema, diseño de estrategias, planteamiento de soluciones, implementación y evaluación de las estrategias de intervención) las y los miembros de la comunidad "aprenden a entender su papel en el proceso de transformación de su realidad social, no como víctimas o como espectadores pasivos, sino como actores centrales en el proceso de cambio" (Balcazar, 2003, p. 62). Así tanto las comunidades como sus integrantes son actores sociales con voz propia y capacidad para analizar y tomar decisiones respecto de su realidad.

Desde la comunicación esto implica la adopción de una perspectiva dialógica. Para Tufte y Mefalopulos (2009) los acercamientos dialógicos implican una comunicación de doble vía, donde tanto el proceso como sus resultados están abiertos y se va más allá de la transmisión de información ya que se genera nuevo conocimiento y soluciones. El equipo de investigación de la UCR adoptó un acercamiento dialógico basado en la horizontalidad en la relaciones y la promoción de la participación para empoderar a las y los vecinos de la comunidad que decidieron involucrarse en el proyecto.

\subsection{La comunidad}

\subsubsection{Luis XV de la Roxana de Pococí}

El perímetro de esta comunidad está definido en buena medida por una gran finca piñera que no solo delimita el espacio comunitario, también se constituye como la primera fuente de trabajo en la zona. Un camino de lastre es la vía principal que atraviesa toda la comunidad. La escuela, una iglesia católica y dos protestantes, varias pulperías, y un bar completan el listado de los espacios públicos en Luis XV.

Se trata de una comunidad rural que, de acuerdo con el último censo, está integrada por 255 hogares. El cultivo de la piña requiere de mano de obra masculina, las jornadas de trabajo son largas e incluyen los sábados. Entre semana y durante el día son las mujeres y los niños los que se observan en la comunidad.

En cuanto a los servicios básicos, hay una escuela primaria. Los jóvenes que estudian deben trasladarse a la comunidad conocida como El Humo (15 minutos a pie) en donde se encuentra el Liceo más cercano. Para la atención básica de la salud, las y los habitantes de Luis XV deben asistir al Ebais (Equipos Básicos de Atención Integral, Ministerio de Salud) que se ubica en la carretera que comunica a Luis XV y El Humo. El servicio de la Fuerza Pública no les presta la atención necesaria y la municipalidad no les brinda el servicio de recolección de residuos sólidos. No tienen instalaciones deportivas ni salón comunal.

Las y los vecinos se organizan en torno a problemas puntuales, de esta forma, tienen varios comités. Por ejemplo, el Comité de Caminos que se crea para velar por que la Municipalidad de Pococí que les asfalte la calle principal. Igual que el Comité de deportes que trabaja para la creación de espacios deportivos y de entretenimiento en la comunidad. Algunas mujeres de la comunidad también están organizadas en torno a alternativas productivas. Es con este grupo de mujeres que establecemos contacto y con quienes iniciamos el trabajo en la comunidad. Ellas manifestaron interés y disponibilidad para desarrollar el proyecto piloto. 
Ambiente limpio, comunidad feliz: análisis de resultados de una campaña de comunicación para la prevención de la fiebre del dengue en las comunidades Luis XV, en Guápiles, Costa Rica

\subsubsection{La encuesta de línea de base: Qué saben las y los vecinos de Luis XV sobre el dengue y su prevención}

Según datos recolectados mediante una encuesta realizada el 18 de octubre del 2013 y administrada en 78 hogares de la comunidad, por el equipo de investigación del proyecto, pudimos indagar acerca de algunos elementos clave para el futuro desarrollo de una campaña comunitaria de comunicación en materia de prevención de dengue.

De las personas encuestadas el $60 \%$ eran amas de casa, no afiliada a organizaciones comunitarias formales. Al igual que el resto de las personas encuestadas manifestaron haber padecido la enfermedad o tener un familiar que ha sido afectado por el dengue (75\%). Esto es indicativo del alto nivel de exposición de las personas de la comunidad a la enfermedad.

Cuando se indagó acerca de la fuente de transmisión de la enfermedad las personas encuestadas en su mayoría $(97,44 \%)$ identificaron al Aedes aegypti, aunque le denominaron de otra manera. Tanto al denominar la enfermedad como su vector, las personas encuestadas hablaban de "dengue". Resulta común, en las entrevistas grupales que las personas se refirieran al mosquito como "dengue", en lugar del nombre científico que se le otorga en las campañas de prevención del Ministerio de Salud.

Las personas encuestadas también poseían conocimientos acertados en relación con los principales entornos que favorecen la reproducción del mosquito (criaderos): aguas estancadas (78\%). Aunque pocas de las personas encuestadas (39\%) reconocieron que el agua debe ser potable.

En términos generales, las personas encuestadas reconocían el reciclaje de llantas y otros materiales como acciones importantes para prevenir la transmisión de la enfermedad pues posibilitan el control del medio ambiente en que se reproduce el vector.

Puede decirse, siguiendo los resultados de esta encuesta que la labor informativa del Ministerio de Salud ha sido efectiva y de importancia para la comunidad.

\subsection{Un antecedente: La campaña "Mi comunidad sin dengue" del Ministerio de Salud}

En el mes de agosto de 2013, ante la emergencia decretada por el aumento de casos de dengue en el país, el Ministerio de Salud lanzó la campaña "Mi comunidad sin dengue" y la movilización social denominada "Día D". El plan de contingencia tuvo como objetivo general "Desarrollar acciones intensivas e integrales de eliminación de criaderos del vector del dengue con participación comunitaria intersectorial para reducir el riesgo y controlar el dengue" (Ministerio de Salud, 2013). En materia de comunicación esta campaña contó con un plan de divulgación que incluyó publicidad, información cara a cara y la elaboración de materiales informativos para las comunidades y la prensa (Ministerio de Salud, 2013). Además propone actividades a nivel comunitario para la eliminación de criaderos, activando las redes institucionales comunitarias (e.g., escuelas, asociaciones de desarrollo, y otras organizaciones no formales).

Una característica de los productos comunicativos de la campaña "Mi comunidad sin dengue" es el estado de contingencia en que se encuentra el país en el mes de 
Ambiente limpio, comunidad feliz: análisis de resultados de una campaña de comunicación para la prevención de la fiebre del dengue en las comunidades Luis XV, en Guápiles, Costa Rica

agosto de 2013 y la necesidad de responder comunicativamente a la contingencia. A pesar de que la comunicación juega un importante papel en los momentos de emergencia en salud, no aparece ningún documento en el que se expliciten las acciones de comunicación o que se exponga una estrategia comunicativa de manera explícita.

A la luz de los insumos que se presentan en la página del Ministerio de Salud y del Informe de Reunión Lucha contra el Dengue (Ministerio de Salud, 2013) no puede hablarse de una estrategia de comunicación sino de la producción de materiales comunicativos de soporte al Plan de Contingencia.

Es importante, discernir entre una campaña de comunicación de prevención del dengue en espacios comunitarios y una campaña de comunicación masiva en contextos de crisis pero nos interesa destacar algunos aprendizajes obtenidos a partir del análisis de la campaña "Mi comunidad sin dengue".

El lenguaje utilizado en los materiales impresos de la campaña tiene como característica fundamental ser directo, concreto y llamar a la movilización. El uso de la primera persona del plural insta a la movilización colectiva y comunitaria en la detección de criaderos. En términos visuales se expresa un elemento clave a tomar en consideración: tanto en los videos, banners y afiches hay un predominio de la representación de objetos y espacios en los que se reproduce el vector, pero poca representación humana en comunidad. Este es un elemento importante, pues como dijimos al inicio el dengue es una enfermedad doméstica y comunitaria. Se da en espacios físicos cuyo nivel de población, o sea, intercambio humano, sea permanente. $Y$ no solo porque la transmisión se de en la relación mosquito-ser humano, sino porque en la interacción humana con el ambiente se construye el habitat de los mosquitos.

Otro elemento que llama la atención es la predominancia de la representación del espacio público sobre el privado. O sea, la representación de la comunidad como espacio abierto: lotes de tierra, calles, espacios comunes es mayor que la representación del espacio doméstico en el que se acumula basura, llantas, recipientes para almacenar agua potable, etc.

En el intercambio con las mujeres de la comunidad Luis XV aprendimos, como equipo de investigación, acerca de la cotidianidad de la prevención y cómo esta labor tiende a ser asumida como parte de las responsabilidades de reproducción de la vida doméstica por parte de las mujeres. Además, hemos aprendido la gran importancia que tiene la educación a niños y niñas en los espacios escolares, tanto en la identificación de larvas y mosquitos, como en el reconocimiento de su habitat y formas de eliminación de las mismas.

A partir de este contacto con la comunidad y los insumos descritos anteriormente, el equipo dio inicio al diseño e aplicación de la Campaña "Ambiente Limpio, Ciudad Feliz"

\section{2. "Ambiente Limpio, Comunidad Feliz"}

\subsection{Proceso participativo de diseño de la campaña}

La campaña de comunicación se diseñó a partir de un proceso de participativo que involucró a las agentes comunitarias de Luis XV, representantes de instituciones ligadas a los temas de dengue y del manejo de residuos sólidos (Área Rectora del Ministerio de Salud de Pococí, Área de Salud de Guápiles-EBAIS, Unidad de 
Ambiente limpio, comunidad feliz: análisis de resultados de una campaña de comunicación para la prevención de la fiebre del dengue en las comunidades Luis XV, en Guápiles, Costa Rica

Gestión Ambiental de la Municipalidad de Pococí y de la Oficina de Supervisión del Circuito Escolar 5 de Guápiles del MEP) y el equipo de investigación de la UCR.

Para ello se realizaron 4 talleres que fueron facilitados por el equipo de investigación de la UCR. En estos talleres se desarrollaron los lineamientos para la identidad visual y se planificó la campaña. Se trató de un proceso interesante en el que el equipo de la UCR adaptó los procedimientos de elaboración de campañas de comunicación para ajustarlos a una dinámica participativa y comunitaria ${ }^{5}$. Desde el punto de vista de la facilitación fue un reto debido a la diversidad de saberes, intereses y grados de implicación de las y los participantes. Por una parte, teníamos a las y los representantes de instituciones quienes están familiarizados con la lógica de planificación de procesos (i.e., elaboración de objetivos, metas e indicadores) y que operan desde los intereses, las posibilidades y limitaciones de las entidades que representan. Y por otra parte, tenemos a las representantes comunitarias quienes no están familiarizadas con la lógica de planificación pero tienen gran claridad sobre lo que necesita la comunidad y ven el espacio de los talleres como una oportunidad para dialogar con las y los representantes de las instituciones sobre los problemas de la comunidad.

Para las y los representantes de instituciones, esta era una iniciativa más entre otras en las que se han participado. Para las mujeres representantes de la comunidad se trataba de un espacio de contacto directo con instituciones y la posibilidad de solucionar un problema importante en la comunidad.

El diseño de la campaña inició con un primer taller el 29 de noviembre del 2013 y finalizó el 27 de febrero del $2014^{6}$. La campaña se implementó en las localidades de Luis XV y El Humo. Los resultados que se presentan en este texto corresponden a la implementación de la campaña en Luis XV.

\subsection{Articulando agendas: Reciclaje - dengue $=$ Comunidad Feliz}

El propósito de la campaña de comunicación surge de la articulación de la agenda de investigación del equipo de la UCR (prevención de la fiebre del dengue en la Roxana de Guápiles) y la priorización de los problemas expresados por las y los vecinos de la comunidad de Luis XV durante el proceso de diagnóstico participativo (mal manejo de los residuos sólidos).

El doble propósito de la campaña queda plasmado en la frase "Ambiente limpio, Comunidad feliz" que se constituye como principal identificador de la iniciativa. El mensaje que las y los participantes desean transmitir es sencillo, la noción de ambiente alude tanto al entorno natural como al entorno social. Según comentaban las y los participantes, las relaciones entre vecinos se habían deteriorado a raíz del mal manejo de los residuos. Ellos pensaban que en la medida en que el ambiente de la comunidad, esté limpio (libre de residuos sólidos y potenciales criaderos del dengue), la comunidad sería un lugar que promueva el bienestar en sus habitantes. Esta forma de concebir una campaña de prevención del dengue se ubica dentro una perspectiva de salud que la concibe no sólo como un estado corpóreo sino que toma

\footnotetext{
${ }^{5}$ Las reflexiones sobre los ajustes al proceso de elaboración de campañas son el objeto de otro artículo.

${ }^{6}$ El proceso se detuvo durante el receso escolar ya que no se contaría con participación de las y los participantes del MEP y las agentes comunitarias que se hacen cargo de hijos y nietos durante las vacaciones.
} 
en cuenta cómo este estado "posibilita la realización de las funciones y, finalmente, de los proyectos vitales, contribuyendo a conformar la sensación de bienestar" (Fernández-López, Fernández-Fidalgo y Cieza, 2010, p. 177). Hasta hace algunos años, las campañas de prevención del dengue en Costa Rica se caracterizaban por darle un gran protagonismo al mosquito y a los síntomas de la enfermedad utilizando un imaginario que ubicaba las acciones preventivas en el plano de la guerra (e.g., La lucha contra el dengue). Desde el 2013, el Ministerio de Salud modifica esta estrategia poniendo el énfasis en los criaderos y no en el mosquito o la enfermedad. En esta estrategia pone en primer plano las acciones comunitarias. Un ejemplo de la nueva estrategia es la iniciativa de "Mi Comunidad sin dengue" (Marroquín y Martínez, 2013).

El equipo que participa del proceso de diseño de la campaña de comunicación se plantea los siguientes objetivos para la campaña:

Objetivo general

Generar consciencia sobre el manejo adecuado de residuos sólidos en grupos específicos de la comunidad de Luis XV para propiciar un cambio en sus hábitos cotidianos relacionados con este manejo que contribuya a la reducción de posibles criaderos de los mosquitos del dengue.

Objetivos específicos

a) Involucrar al menos a un $10 \%$ de hogares en cada una de las comunidades en actividades relacionadas con el reciclaje para mantener los hogares libres de posibles criaderos.

b) Formar al menos 40 agentes comunitarios que trabajen en la diseminación de información y las practicas vinculadas al manejo adecuado de residuos sólidos y de criaderos del dengue en ambas comunidades.

c) Modificar la percepción de las amas de casa y los niños que participen en los Talleres de Mi Casa Verde con respecto a los residuos sólidos, para que los vean como materiales a los que se les puede dar otro uso o bien reciclarlos para que no se conviertan en criaderos del mosquito del dengue.

En este sentido, la campaña se concentra en generar consciencia para la eliminación de posibles criaderos del dengue a partir de un manejo adecuado de los residuos sólidos en la comunidad. Los públicos a los que va dirigida la campaña son amas de casa y niños en edad escolar.

Los mensajes clave utilizados en la campaña fueron:

- Los residuos sólidos mal dispuestos pueden convertirse en criaderos del mosquito de dengue.

- Reduzca su bolsa de basura, reciclando. No todo lo que se desecha es basura, hay residuos sólidos que se pueden reciclar o transformar para darles un nuevo uso.

- Reciclar reduce mi bolsa de basura y me ayuda a eliminar potenciales criaderos.

Para cumplir con los objetivos planteados se desarrollan las siguientes actividades como parte de la campaña: 
Ambiente limpio, comunidad feliz: análisis de resultados de una campaña de comunicación para la prevención de la fiebre del dengue en las comunidades Luis XV, en Guápiles, Costa Rica

a. Sistema de recolección de reciclaje: se coordinó con una persona para la recolección gratuita y quincenal de residuos reciclables. Se elaboró un material sencillo sobre los tipos de residuos y cómo separarlos para el reciclaje. Este material se distribuyó casa por casa en la actividad del "Puerta a Puerta" que se detalla más adelante.

b. Festival "Ambiente y Salud": El lanzamiento de la campaña se hace por medio de una actividad lúdica y educativa dirigida a las familias de la comunidad de Luis XV. Los mensajes clave de la campaña se entregaron de diversas formas a lo largo del festival. Por ejemplo, se creó una obra de teatro para ilustrar los problemas que experimenta la comunidad a raíz del mal manejo de los residuos sólidos y explorar soluciones. La Colectiva Respiral $^{7}$ estuvo a cargo del guion y la puesta en escena de la obra. Se montaron puestos informativos sobre los criaderos del dengue, el cuido del medio ambiente y el uso de materiales reciclables para manualidades. Además se dispuso de recipientes debidamente identificados para separar los residuos sólidos que se generarían durante la actividad. Un grupo de voluntarios se mantuvo cerca de los recipientes para explicar a las personas cómo separar los residuos según su composición.

c. Puerta a Puerta: visitas cortas a los hogares de ambas comunidades para explicarles el servicio de reciclaje y cómo reciclar. También se aprovechó para invitar a los Talleres de "Mi Casa Verde". Un equipo compuesto el grupo de agentes comunitarias, el equipo de investigadores y estudiantes de la $\mathrm{UCR}^{8}$ se encargaron de realizar las visitas.

d. Talleres de "Mi Casa Verde": espacio semanal para capacitar a las y los miembros de la comunidad en los temas que le permitan transformar su hogar en una casa verde. Se plantearon tres talleres cada uno de ellos abordaría un tema (reciclaje, ambiente y salud; manejo de criaderos del dengue y el chikungunya, alternativas para el manejo de residuos no reciclables). El eje trasversal sería el reciclaje y transformación de residuos sólidos en objetos decorativos de utilidad cotidiana. Los talleres serían facilitados por las representantes comunitarias y una persona del equipo de investigación.

Como se puede notar la campaña se apoya fuertemente en la comunicación de pares para transmitir los mensajes, por tanto, no se trata de una estrategia masiva, sino más bien de una estrategia dirigida a grupos pequeños que se encargaran posteriormente de difundir el mensaje a otros. Se parte de la idea de fomento de capacidades para empoderar a la comunidad para que ellos mismos sean capaces de sostener y darle mayor difusión al proceso.

\subsection{Resultados obtenidos}

\footnotetext{
${ }^{7}$ El trabajo de Respiral articula el teatro del oprimido (Augusto Boal) y el teatro foro clown. El teatro del oprimido concibe a la obra como un recurso para el cambio social, en la puesta en escena se evidencian las asimetrías e injusticias de las cuales son objeto las y los participantes. No se trata solo denunciar sino que las y los participantes utilicen el espacio de la obra para modificar aquello que oprime y que genera malestar. En este sentido, las y los participantes no son simples espectadores sino que pueden convertirse en agentes de cambio, protagonistas de su propia historia. La técnica del foro clown, utiliza el recurso del payaso para abordar temas y situaciones delicadas desde el humor.

${ }^{8}$ De la Escuela de Ciencias de la Comunicación Colectiva así como las y los estudiantes del TCU Prácticas Saludables.
} 
Las campañas de comunicación en el campo de comunicación y salud tienden a evaluarse cuantitativamente en función de su impacto en la población meta. El impacto generalmente se mide a nivel individual y reporta el cambio en conocimientos, actitudes o comportamientos. Esta medición se hace a partir de datos estadísticos que comparan el grado de conocimiento, las actitudes y las percepciones antes y después de la intervención o campaña. También se considera la cantidad de participantes que adoptaron la innovación, la práctica o el hábito para demostrar el cambio en comportamiento. Tufte y Mefalopulos (2009) señalan que este tipo de mediciones tienden a dejar de lado aspectos como la satisfacción y las percepciones de las y los participantes con respecto al cambio adoptado. Este tipo de datos se obtienen a partir de metodologías cualitativas que permiten explorar el mundo subjetivo de las y los participantes.

Tomando en cuenta esta dicotomía en los modos de evaluación de campañas de comunicación y valorando los vacíos que presentan, decidimos adoptar una metodología mixta para la evaluación de la campaña Ambiente Limpio, Comunidad Feliz. Por una parte, aplicamos un cuestionario de Conocimiento, Actitudes y Percepciones con respecto al Dengue para medir la incidencia de la campaña en el nivel de conocimiento, actitudes y percepciones de la comunidad con respecto al dengue. A este cuestionario se adicionó una sección que evaluó la recordación de la campaña y sus mensajes clave así como la adopción del hábito de reciclaje. Por otra parte, se realizaron nueve entrevistas semi-estructuradas con las representantes comunitarias y se organizó un grupo focal con personas que participaron en los Talleres de Mi Casa Verde. El objetivo era identificar aquellos aspectos más valorados por las y los participantes y aquellos por mejorar. También nos interesaba conocer cómo habían interpretado los mensajes de la campaña y qué ideas tenían para difundirla a más personas y darle sostenibilidad en el tiempo.

\subsubsection{Evaluación cuantitativa}

El modelo RE-AIM (por sus siglas en inglés) define 5 dimensiones para la medición del impacto de los programas de promoción de la salud (Glasgow, Klesges, Dzewaltowski, Estabrooks y Vogt, 2006). Estas dimensiones resultan muy útiles para comprender de la complejidad de la variable de impacto en una intervención. Las cinco dimensiones son: i) El alcance que corresponde al porcentaje y representatividad de individuos dispuestos a participar; ii) La efectividad se refiere al impacto de la intervención en los resultados esperados y la calidad de vida del grupo beneficiario; iii) la adopción y la implementación parten de la premisa que las intervenciones se desarrollan desde instituciones u organizaciones formales. La adopción se refiere al porcentaje de representantes y personal que aceden a desarrollar la iniciativa e implementación rinde cuenta de la consistencia con la que los distintos actores desarrollan la iniciativa en distintos lugares. Por último, iv) el mantenimiento está relacionado con la sostenibilidad del proyecto y el grado en que las y los participantes individuales mantienen el cambio de comportamiento a largo plazo. 
Ambiente limpio, comunidad feliz: análisis de resultados de una campaña de comunicación para la prevención de la fiebre del dengue en las comunidades Luis XV, en Guápiles, Costa Rica

\subsubsection{Alcance y efectividad de la campaña}

En cuanto al nivel de recordación e identificación de la campaña un $56 \%$ de los hogares encuestados había oído hablar de la campaña Ambiente Limpio, Comunidad Feliz. De este porcentaje de hogares, una tercera parte $(36 \%)$ había participado en las actividades de la campaña. La mayoría (94\%) participó en el Festival de Lanzamiento de la Campaña, seguido por un $72 \%$ que reportan haber asistido a los talleres y poco más de la mitad (56\%) manifestaron reciclar. En general, los hogares encuestados lograron captar una parte del mensaje de la campaña. Así el $44 \%$ de los hogares consideró que la campaña trataba sobre el reciclaje mientras que un $38 \%$ captó la esencia de la campaña, que consistía en la reducción de criaderos del dengue a través del reciclaje.

El nivel de recordación e identificación de la campaña resulta menor (56\%) al alcance de la campaña en términos de los hogares que reportan conocer el sistema quincenal de recolección gratuita de residuos reciclables (86\%), uno de los componentes más importantes de la campaña. En cuanto a la efectividad de la campaña de acuerdo con los objetivos planteados podemos constatar que $59 \%$ de los hogares que reportan reciclar han utilizado el servicio de recolección de reciclaje al menos una vez. El objetivo de la campaña era involucrar un $10 \%$ de hogares en actividades de reciclaje en cada comunidad. El $59 \%$ corresponde a un $18 \%$ del total de hogares en Luis $\mathrm{XV}^{9}$. Casi se dobla la meta establecida. La campaña se planteó la formación de al menos 40 agentes comunitarios en cada comunidad a partir de los Talleres de Mi Casa Verde. La asistencia a los Talleres de Mi Casa verde en Luis XV fue poco constante, se crearon dos grupos. El primer grupo empezó con 23 personas, de las cuales sólo 6 lograron cumplir con la asistencia a los tres talleres. El grupo 2 arrancó con 13 personas, de las cuales tan sólo 3 asistieron a los tres talleres. En Luis XV se logró formar a 9 personas mientras que en El Humo se formaron 12 para un total de 21 la mitad de lo que nos habíamos planteado en el objetivo.

Desde esta perspectiva de evaluación hay espacio para mejorar los niveles de recordación de la campaña en sí a la vez que señala el bajo rendimiento de los talleres como estrategia para reclutar nuevas y nuevos agentes comunitarios.

\subsubsection{Evaluación cualitativa}

En esta evaluación (entrevistas semi-dirigidas y grupos focales) se destacan los cambios percibidos por las y los participantes en la comunidad a raíz del proyecto. Se mencionan los principales aprendizajes y aquello que se puede mejorar.

\subsubsection{Cambios percibidos en la comunidad}

Las personas entrevistadas perciben casi en forma unánime que la comunidad está más limpia. Por ejemplo, Nieves menciona "La comunidad si ha cambiado mucho, porque antes había basura por donde quiera que usted veía, y desde que nos vinieron a ayudar a hacer eso, las calles se ven mucho más limpias. Ha sido un

\footnotetext{
${ }^{9}$ Según el censo 2011 del INEC Luis XV tiene un total de 255 viviendas. El 59\% de hogares que han usado el servicio de reciclaje más de una vez corresponde a 46 hogares.
} 
Ambiente limpio, comunidad feliz: análisis de resultados de una campaña de comunicación para la prevención de la fiebre del dengue en las comunidades Luis XV, en Guápiles, Costa Rica

cambio bastante grande, antes usted pasaba por aquí y eran miles de bolsas de Picaritas y Meneitos y ahora no. Todo está más limpio" (entrevista, fecha). En la misma línea Lidieth comenta "Porque antes el pueblo, antes estaba, esos ríos que son una quebradita antes estaban llenos de basura. Allá frente de mi casa a un costado ahí había un basurero municipal... y hoy ya no está. Entonces se ha logrado mucho" (entrevista, fecha).

Algunas agentes comunitarias perciben un cambio de actitud hacia el manejo de los residuos que se manifiesta principalmente en la buena disposición hacia la práctica de reciclaje "mucha gente, ellos ya no queman, ya aprendieron ...como dicen a reciclar, entonces ya uno así evita ver basura tirada por todo lado" (Marielos, entrevista, fecha).

La mayoría de las personas entrevistadas perciben una disminución en los casos de dengue en la comunidad. Lidieth recuerda que cuando inició el proyecto (julio 2012) había mucho dengue, "ellos [equipo de investigación de la UCR] venían con esa expectativa de controlar la enfermedad" (entrevista, fecha). Marielos comparte esta percepción "yo creo que ha bajado, ya que hace unos dos o tres años era un montón de dengue y ahora no es que no aparecen casos, pero si se ha mermado un montón, entonces yo sí creo que a la gente sí le ha llegado un poco el mensaje" (entrevista, fecha).

Otro impacto que ligan al proyecto y la campaña es que las y los vecinos tienen más contacto y relación entre ellos, Mitcina considera que antes "No nos comunicábamos tanto, era muy aparte, ellas en su casa y yo en mi casa" (entrevista, fecha). La percepción de Lidieth es similar para ella la relación con las y los vecinos "ha cambiado a tener más comunión con el pueblo, más participación, nos hemos unido más" (entrevista, fecha). Martín, otro representante comunitario, nos indicó que antes del proyecto y la campaña había "menos organización de la gente" él considera que la campaña "Ha ayudado a organizar, hay mucha gente que está reciclando" (entrevista, fecha). Marielos considera que el tener más contacto a contribuido a organizar a la gente en torno a otros problemas de la comunidad, por ejemplo, el arreglo de la calle principal Luis XV. Las y los miembros de la comunidad que se organizaron y lograron que la Municipalidad aplanara y aplicara lastre a la calle.

\subsubsection{Sostenibilidad}

En menos de dos meses se cumplirá un año desde que finalizó la campaña "Ambiente Limpio, Comunidad Feliz" y podemos decir que la iniciativa continua y es sostenida por las representantes de la comunidad y el grupo de los niños ecologistas de El Humo. El sistema de reciclaje se ha mantenido en la comunidad. Las responsables le han hecho algunas modificaciones, por ejemplo, el camión ya no pasa casa por casa, sino que hay puntos de acopio en donde el camión recoge. En cuanto a los talleres en temas de reciclaje y dengue, los ha asumido el grupo de Los niños ecologistas que organizan concursos y talleres con cierta regularidad.

\section{REFLEXIONES FINALES}

Las metodologías IPbC e IAP resultaron idóneas para trabajar el proceso de participación ligado a la salud en las comunidades mencionadas, ya que la promoción de horizontalidad en las relaciones entre participantes y el valor que se le 
da a los saberes locales contribuyen al empoderamiento de las personas que participan en el proceso. Un aspecto que fue difícil lograr está relacionado con el grado participación de las agentes comunitarias en el proceso de investigación. Las participantes no lograron asumir un rol de mayor empoderamiento con respecto a los procesos de investigación, se llegó a una participación de tipo consulta, a pesar de que se trabajó de forma tal que ellas pudieran tener una mayor influencia en los procesos desarrollados. Esto fue más evidente en el desarrollo del plan de comunicación, y en la elaboración de los materiales de comunicación. Sin embargo, el empoderamiento se manifestó en una serie de acciones que las agentes comunitarias emprendieron por ellas mismas para gestionar la resolución de otros problemas que experimenta la comunidad. Por ejemplo, se organizaron para manifestarse en contra de la eliminación de la farmacia del Ebais de San Antonio, también elaboraron una carta, recogieron firmas y solicitaron audiencia con el Alcalde la Municipalidad de Pococí para gestionar la instalación de alcantarillas y la pavimentación de la calle principal que es de lastre. El Alcalde las atendió y realizó en estos días una visita y reunión con representantes de la comunidad.

Los procesos de participación comunitaria son procesos voluntarios que se nutren del tiempo que le puedan consagrar las y los interesados, es decir, dicho proceso es paralelo a las ocupaciones (afuera a adentro del hogar) y las agendas de las personas que participan. En este proyecto la mayor parte de las participantes son mujeres, amas de casa. Las que se involucraron más y de forma sostenida en el proyecto fueron aquellas que tenían una situación económica estable y cuyas hijas e hijos eran adolescentes o adultos. Ambos factores redundan en la posibilidad de dedicar más tiempo a actividades adicionales a su trabajo de amas de casa. El fenómeno que se percibe en términos de participación se puede ilustrar a través de dos círculos concéntricos, donde uno es el núcleo compuesto por las personas que desean y pueden implicarse más y el otro conformado por personas que están en la periferia que se implican de acuerdo a su interés y posibilidades. Este último círculo no está tan bien definido como el otro y fluctúa considerablemente. El problema con las personas del núcleo es que se cansan, para darle sostenibilidad al núcleo debería de darse una especie de relevo por parte de las personas de la periferia, situación que además le daría una mayor estabilidad al círculo externo.

Finalmente, el dengue es una experiencia que las y los vecinos de Luis XV han vivido en carne propia o bien de muy cerca, esto ha hecho que lo vean como algo común y sobre todo como algo que no es mortal. El sentido de alerta ante la enfermedad ha decrecido y las personas se van a movilizar en torno al dengue en el momento en el que se presenta un brote o epidemia. Es por ello que resultó crucial ligar el dengue a otro problema que les apele más y sobre todo que tenga una incidencia cotidiana, en este caso fue el manejo de residuos sólidos, problema que resultaba crucial para la comunidad y que además está muy relacionado con las rutinas de vigilancia de criaderos potenciales del dengue.

\section{REFERENCIAS}

Balcázar, F. (2003) Investigación acción participativa (iap): Aspectos conceptuales y dificultades de implementación. Fundamentos en humanidades, Año IV - $\mathrm{N}^{\circ}$ I/II (7/8), 59-77. 
Calderón-Arguedas, O., Troyo, A., Solano, M. E., Avendaño, A., Beier, J. (2009) Urban mosquito species (Diptera: Culicidae) of dengue endemic communities in the Greater Puntarenas Area, Costa Rica. Revista de Biología Tropical, 57(4), 1223-1234.

Fals Borda, O., Bonilla, V., Castillo, G. Libreros, A. (1972) Causa Popular, Ciencia Popular. Bogotá: La Rosca.

Fernández-Lopez, J. A., Fernandez-Fidalgo, M., \& Cieza, A. (2010). Los conceptos de calidad de vida, salud y bienestar analizados desde la perspectiva de la clasificación internacional del funcionamiento (CIF). Revista Española de Salud Pública, 84(2), 169-184.

Glasgow, R. E. (2006). Evaluating the impact of health promotion programs: using the RE-AIM framework to form summary measures for decision making involving complex issues. Health Education Research, 21(5), 688-694. doi:10.1093/her/cyl081

S. Lazarus, B. Duran, L. Caldwell and S. Bulbulia (2012). Public Health Research and Action: Reflections on Challenges and Possibilities of Community-Based Participatory Research, En J. Maddock (Ed.). Public Health - Social and Behavioral Health (pp. 309-324). Croacia: InTech

Marroquín, L. y Martínez, Y. (2013) Análisis de contexto para la elaboración de una estrategia comunitaria de comunicación para la prevención del dengue en la comunidad Luis XV. En: CICOM (2013) Memoria II Mini-Jornadas de Investigación (pp.113-129). Universidad de Costa Rica.

Ministerio de Salud (2013) Materiales educativos para campaña Mi comunidad sin dengue. Recuperado de http://www.ministeriodesalud.go.cr/index.php/centrode-informacion/material-educativo/cat_view/56-centro-de-informacion/58material-educativo/585-materiales-dengue-dia-d.

Quintero, J., Brochero, H., Manrique-Saide, P., Barrera-Pérez, M., Basso, C., Romero, S., ... Petzold, M. (2014). Ecological, biological and social dimensions of dengue vector breeding in five urban settings of Latin America: a multi-country study. BMC Infectious Diseases, 14(38). doi:10.1186/1471-2334-14-38

Shepard, D. S., Coudeville, L., Halasa, Y. a., Zambrano, B., \& Dayan, G. H. (2011). Economic Impact of Dengue Illness in the Americas. American Journal of Tropical Medicine and Hygiene, 84(2), 200-207. doi:10.4269/ajtmh.2011.100503

Tufte, T., \& Mefalopulos, P. (2009). Participatory Communication: A Practical Guide. Washington, DC.: World Bank. 